\title{
Effects of Bacillus subtilis and Bacillus licheniformis on catfish in industrial aquaculture
}

\author{
Elena Romanova ${ }^{1, *}$,Elena Spirina ${ }^{1}$, Vasily Romanov ${ }^{1}$, Vaselina Lyubomirova ${ }^{1}$, and \\ Ludmila Shadyeva ${ }^{1}$ \\ ${ }^{1}$ State Agrarian University named after P.A., Stolypin, 1, Novy Venets Blvd., 432017, Ulyanovsk, \\ Russia
}

\begin{abstract}
When growing fish in high-tech breeding systems, it is exposed to many stress factors. To reduce stress, we used spore forms of microorganisms - Bacillus subtilis and Bacillus licheniformis, possessing probiotic properties. Two groups were formed, a control group and an experimental group that received probiotic microorganisms with feed. The aim of the study was to evaluate the effectiveness of the use of microorganisms - Bacillus subtilis and Bacillus licheniformis to reduce stress in fishes. For this indicator points were selected - stress hormone cortisol and antioxidant defense enzymes. The research results showed that stress level assessed by cortisol in fishes fed with probiotic microorganisms is 2.8 times lower than in the control group. The use of micronucleus test to assess cytogenetic damage showed that against the probiotic microbiota background, the number of cells with fragmented nuclei decreases by 5.7 times. Antioxidant defense enzyme activity when using Bacillus subtilis and Bacillus licheniformis increased, indicating activation of the defense system against oxidative stress. The obtained results demonstrated the high efficiency of the use of spore forms Bacillus subtilis and Bacillus licheniformis when growing catfish in high-tech fish farming systems. The Russian Foundation for Basic Research has supported our study with the grant No. 18-016-00127.
\end{abstract}

\section{Introduction}

Today, industrial aquaculture is becoming more and more focused on high-tech fish farming systems, which are considered the top of modern fish farming. Industry progress has become a knowledge-based process. It is provided by innovative technologies that increase the efficiency and profitability of fish farming.

Today, aquaculture is one of the rapidly growing sectors of agriculture. Its progress allows to increase the production of marketable products, primarily due to the optimization of hydrochemical regime parameters, feed composition and the use of probiotics $[1,2]$.

Probiotics in agricultural production appeared in connection with the development of biotechnology for the microbial preparations production. Recently, in Russia and abroad, it

\footnotetext{
* Corresponding author: vvr-emr@yandex.ru
} 
began to get a lot of attention. The problem of the effective use of probiotics in aquaculture has become urgent.

There is every reason to suppose that the use of probiotics in aquaculture is an effective way to increase its productivity and the quality of farmed products [3, 4]. Probiotics not only increase the biomass growth rate, but also increase the ecological purity of fish as a food product.

In modern conditions, as an object of industrial fish farming, it is becoming increasingly popular the catfish (Clarias gariepinus). This fish species has a very high growth rate and surpasses all 49 fish species grown in Russian industrial aquaculture by this indicator. [1, 2]. Catfish has a high palatability and is considered to be delicious. According to the chemical composition and content of essential amino acids, omega 3 fatty acids catfish meat can be attributed to functional foods.

Growing fish in industrial aquaculture, the highest form of which is the installation of closed water supply (CWS), creates a number of problems that need to be resolved. One of such problems is the state of chronic stress experienced by the fish throughout the growing period [5-7]. First of all, this stress is caused by high planting densities, oxygen deficiency, manipulations during preventive measures, sorting, control weighings, transplantation, etc. [5-7] Under conditions of stress, increased secretion of catecholamines, such as adrenaline and noradrenaline, as well as cortisol, occurs in the fish organism. [8]. It is known that a high cortisol content suppresses the immune system [9], activates the processes of catabolism, and affects the course of mitotic division of hematopoietic cells [8].

In our work, we examined the level of cortisol, which was considered as stress indicator. [8]. The negative effect of cortisol on the cell was evaluated by the micronucleus test $[10,11]$, which was carried out in order to reveal the damaging effect of the hormone cortisol on the mitotic process. Fish erythrocytes were used as a model for assessing the effect of cortisol on cells.

The activity of antioxidant defense enzymes catalase and peroxidase were used as informative indicators of oxidative stress. Under conditions of stress, an increase in the activity of enzymes occurs in the fish organism, providing lipid oxidation in mitochondria for ATP synthesis [5,6]. The consequence of oxidative stress is the formation of free radicals that damage biomolecules and cause its functions violation. This leads to numerous morphological and functional changes in the fish organism, causes homeostasis violation and leads to pathology development $[5,6]$.

To minimize the damage from chronic stress effects on the fish organism, to increase adaptive plasticity by enriching it with enzymes, essential amino acids and vitamins, we used probiotics. The purpose of probiotics is to heal the intestinal microbiocenosis of fish and increase the immunity $[11,12]$. Breeding in the intestines, probiotic bacteria produce biologically active substances that stimulate the immune system, increase feed conversion and fish growth rate $[11,12]$.

The used sporothermin probiotic is based on the spore forms of bacterial strains Bacillus subtilis and Bacillus licheniformis. The use of spore forms allows microorganisms to survive under the influence of stomach acidic environment and under bile action.

The indicator of the live bacteria content in the preparation is CFU - the number of colonies forming units. In the sporotherm the content of Bacillus subtilis and Bacillus licheniformis no less than $5^{10} \mathrm{CFU} / \mathrm{g}$. The content of own microbiota in the fish intestines is four orders of magnitude lower. Therefore, in the fish intestines under the influence of probiotic microorganisms, the dominance phenomenon is realized, which consists of the fact that the probiotic microbiota suppresses the pathogenic and conditionally pathogenic microbiota of the intestinal microbiocenosis, regulating its structure [13].

Bacterial strains Bacillus subtilis and Bacillus licheniformis have pronounced antagonistic activity to a wide range of pathogenic and opportunistic microorganisms [14]. 
The aim of the study was to evaluate the effectiveness of using microorganisms Bacillus subtilis and Bacillus licheniformis to reduce stress in fishes.

\section{Materials and methods}

The object of the study was catfish (Clarias gariepinus), grown in closed water supply (CWS). One of the pools contained fish, which received a balanced feed company Coppens, the second contained fish, which was fed compound feed of the company Coppens, which was modified ourselves with the addition of the probiotic sporothermin at a dose of $4 \mathrm{~g}$ per $1 \mathrm{~kg}$ of feed, for three months. The probiotic known under the brand name sporothermin consists of spore- and heat-resistant forms of microorganisms Bacillus subtilis and Bacillus licheniformis, the content of which in the chemical is not less than $5^{10}$ colony forming units per $1 \mathrm{~g}$ of the drug.

The studies were carried out at the faculty of veterinary medicine and biotechnology of the Ulyanovsk State Agrarian University. Water temperature in the pools was maintained at $26^{\circ} \mathrm{C}$, the oxygen content in water was $4 \mathrm{mg} / \mathrm{l}$.

Blood sampling from catfish was carried out intravitally by decapitation into tubes containing an anticoagulant, then the tubes were centrifuged for 7 minutes at $3000 \mathrm{rpm}$, the plasma was stored at a temperature minus $12-18^{\circ} \mathrm{C}$. The cortisol concentration was determined according to the manufacturer's instructions, using commercial kits for enzymelinked immunosorbent assay (Hema, Russia).

The micronuclear test was performed on smears of peripheral blood, scanning at least 2000 erythroid cells. Catfish blood smears were fixed in the May-Grunwald fixative, stained with Romanovsky-Giemsa dye [10]. Round chromatinic body with a continuous smooth edge lying separately from the nucleus, but in the same plane with it, having the same intensity color and the same chromatin pattern, was taken as a micronucleus. Determination of micronuclei in catfish peripheral blood was performed according to the criteria of J. Merch [11].

The activity of enzymes of the antioxidant system was determined (units / mg protein) on a two-beam spectrophotometer UV-1800 (Shimadzu, Japan) wavelength $\lambda=480 \mathrm{~nm}$ and $\lambda=347 \mathrm{~nm}$, at room temperature.

Catalase activity was determined by the decomposition of hydrogen peroxide [15-16]. Activity superoxide dismutase (SOD) determined in the system nitro-blue tetrazolium phenazinemethosulfate-nicotinamidedinucleotide (NBT-PMS-NADN). Activity was expressed in nmol of substrate / min per $\mathrm{mg}$ of protein. Statistical processing of the obtained data was performed using the application package «STATISTICA-6». The differences were considered statistically significant at $\mathrm{p}=0.05$.

The main statistical characteristics of the studied parameters were determined. ( $\bar{X}$, $\mathrm{Me}, \mathrm{D})$. The distribution was nonparametric in most of the studied parameters; nonparametric methods were used to analyze the differences between independent samples (Wilcoxon's uniformity criterion - U).

\section{Results}

At the first stage of the research, the content of cortisol in the catfish blood grown in industrial aquaculture was determined. The results of the studies have showen that the cortisol content in catfish grown in closed water supply plants is high and amounts to 23.8 $\pm 0.9 \mathrm{nmol} / \mathrm{l}$, (fig. 1). This is a high indicator of high stress levels in fishes. 
At the next work stage, the cortisol content the peripheral blood of catfish grown using the probiotic sporothermin was estimated. According to the results of the research, it amounted to $8.4 \pm 0.1 \mathrm{nmol} / 1$ (fig. 1).

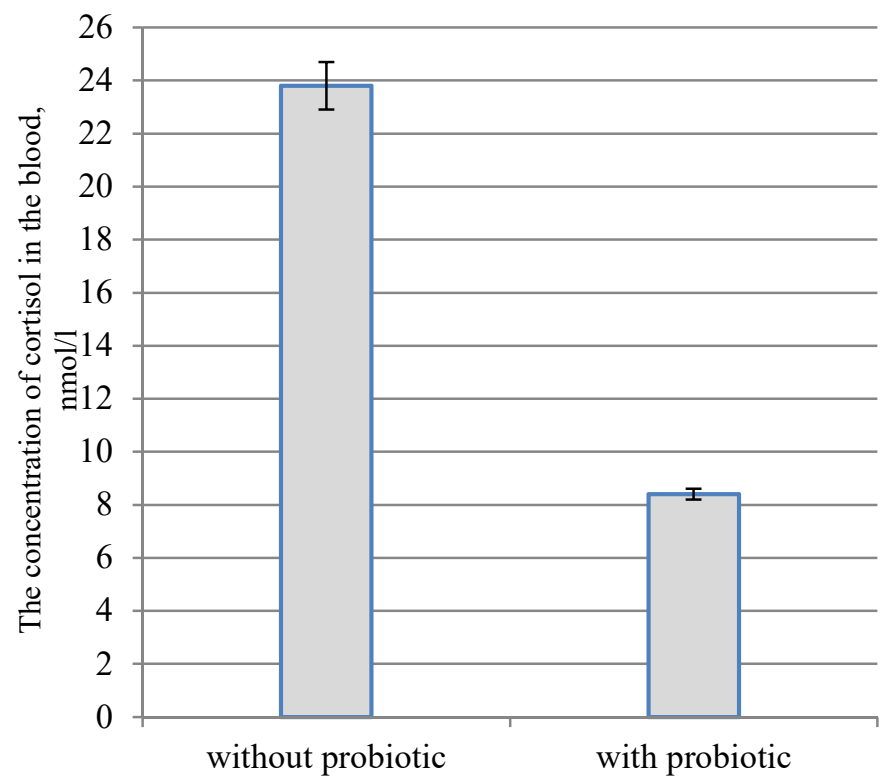

Fig. 1. The level of cortisol in catfish (Clarias gariepinus) when grown in high-tech industrial aquaculture.

Analyzing the obtained results, it should be noted that a significant excess of cortisol levels was found in catfish blood compared to its content in fishes grown using feed containing probiotic microorganisms. Against the background of using spore forms Bacillus subtilis and Bacillus licheniformis the cortisol level in the fish blood was 2.8 times lower.

It was noted above that the stress hormone cortisol in high concentrations is capable of exerting a cytotoxic effect. Cortisol is able to disrupt the process of mitosis, destabilizing the cell nuclear apparatus. This hormone can cause fragmentation of the nucleus, accompanied by damage to the genetic material $[13,14]$ and the formation of micronuclei.

Based on the foregoing, at the next research stage we conducted a micronuclear test. It was carried out by direct counting of catfish peripheral blood erythrocytes, that had damage to the nuclear apparatus with the release of micronuclei. Three types of micronuclei were found in the studied individuals, which were accordingly classified as A, B, C.

In the peripheral blood erythrocytes, the proportion of cells with micronuclei of the type «A» composed $4.9 \pm 1.4 \%$, with micronuclei of type «B» $-3.1 \pm 1.5 \%$, of type «C» $2.9 \pm 0.5 \%$ (fig. 2). In general, the level of red blood cells that had micronuclei in fishes grown without probiotic microorganisms was $10.9 \%$.

To disorders of erythrocyte nuclei of type «A» pertained micronuclei that are so isolated that had lacked communication with the nucleus; to pathology of type «B» pertained micronuclei, closely adjacent to the nucleus and having a structure and color corresponding to the cell nucleus; to pathology of type «C» pertained changes in the nuclear apparatus, accompanied by the appearance of large micronuclei, the diameter of which varied from one tenth to one third of the diameter of erythrocyte nucleus [11]. 


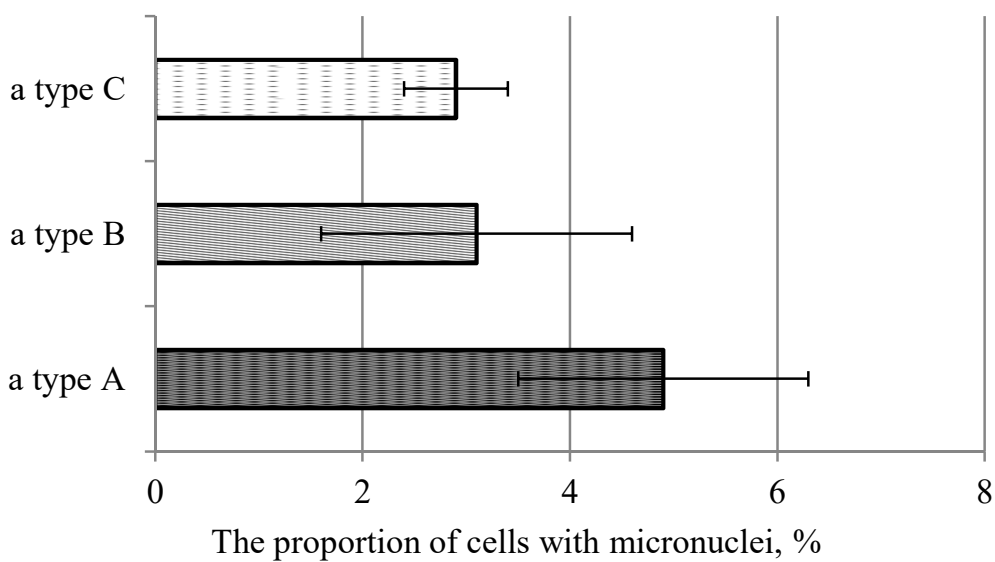

Fig. 2. The proportion of cells with micronuclei when grown in high-tech industrial aquaculture without probiotic.

Completely different situation was observed in peripheral blood erythrocytes of fish grown using spore forms Bacillus subtilis and Bacillus licheniformis; its proportion of erythroid cells with micronuclei of type A composed $1.9 \pm 1.1 \%$ (fig. 3). No other types of core pathology were detected in this group of fish. The difference is obvious, in fishes treated with probiotic, nuclear apparatus damage was 5.7 times less.

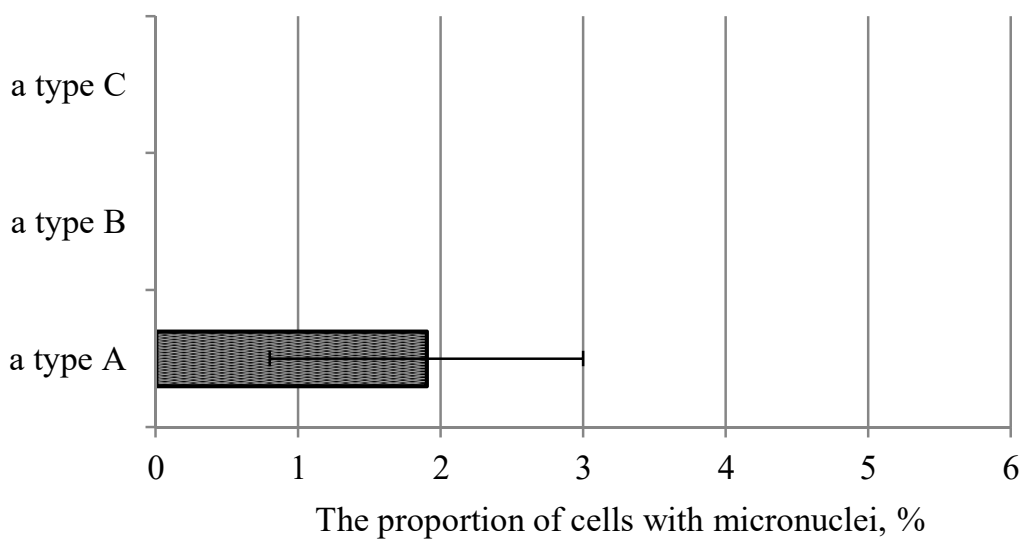

Fig. 3. The proportion of cells with micronuclei when grown in high-tech industrial aquaculture with probiotic.

The calculation of the percentage of peripheral blood erythrocytes that had micronuclei showed that there is a significant positive effect of cultures of microorganisms of the probiotic sporothermine on the catfish erythrocyte cytogenetic homeostasis.

An important indicator of the oxidative stress level is the antioxidant protection state. Therefore, at the next work stage, we studied the activity of catalase and superoxide dismutase enzymes in erythrocytes and blood serum of catfish.

The complex mechanism of antioxidant defense of the organism includes the following enzyme systems as the main components: superoxide dismutase, catalase, glutathione reductase, glutathione peroxidase and glutathione transferase [17-18]. 
The main role in the protection mechanism against oxidative stress is given to catalase, which has a pronounced antioxidant effect [19-21]. Catalase, like superoxide dismutase, protects the organism from the inevitably formed highly toxic oxygen radicals [22-24].

The superoxide dismutase and catalase activity were determined in blood plasma and erythrocytes in fishes. The activity of these enzymes was studied in fishes grown on traditional compound feeds of the Coppens company and on compound feeds of the same company with the addition of probiotic.

The results of determining the antioxidant enzymes activity: catalase and superoxide dismutase in blood and in erythrocytes of catfish are shown at fig. 4 and 5 .

It was found that using traditional Coppens feeds, catalase activity in blood serum of catfish grown in CWS was $0.9 \pm 0.03 \mathrm{nmol}$ of substrate / min per $\mathrm{mg}$ of protein, and in erythrocytes it was significantly higher and amounted to $1.37 \pm 0.8 \mathrm{nmol}$ of substrate $/ \mathrm{min}$ per mg squirrel (fig. 4)

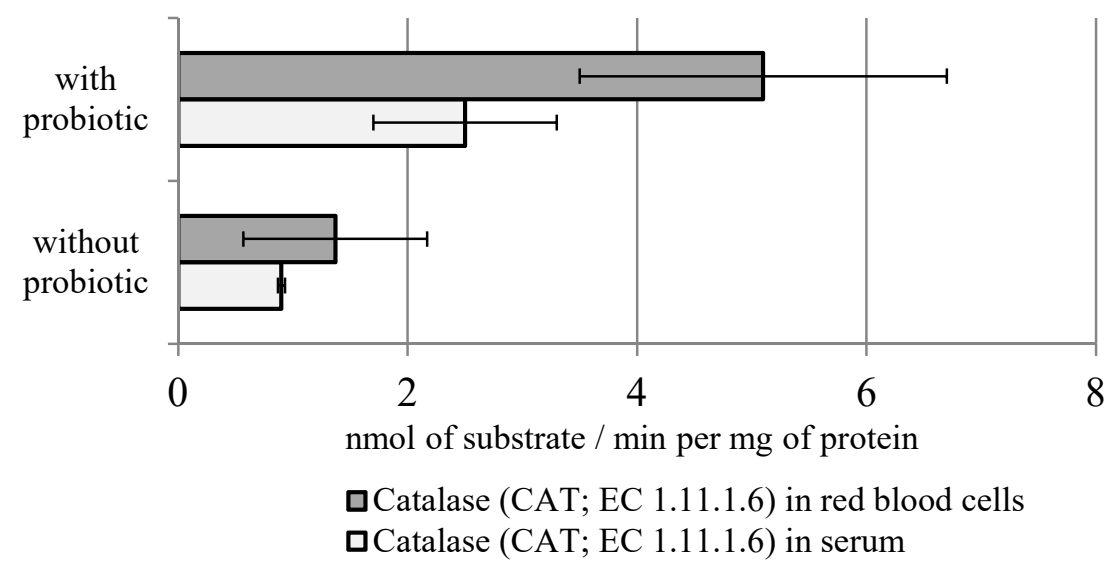

Fig. 4. The activity of catalase in serum and red blood cells of catfish.

In catfish grown on feed containing spore forms of microorganisms Bacillus subtilis and Bacillus licheniformis, antioxidant defense enzyme activity was different (fig. 4).

The catalase activity in the blood serum of catfish treated with the probiotic was significantly higher and reached $2.5 \mathrm{nmol}$ of substrate / min per $\mathrm{mg}$ of protein, i.e. increased 2.7 times.

Increasing catalase activity in erythrocytes of fish grown on feed containing spore forms of microorganisms Bacillus subtilis and Bacillus licheniformis as part of the probiotic sporothermin was even more pronounced. Catalase activity increased by 3.7 times and amounted to $5.1 \pm 1.6 \mathrm{nmol}$ of substrate / min per mg of protein.

The activity of superoxide dismutase in the blood serum of catfish grown on probioticfree feeds was $178.7 \mathrm{nmol}$ of substrate / min per mg of protein, and in erythrocytes $245.2 \pm$ $11.8 \mathrm{nmol}$ of substrate / min per mg of protein (fig. 5). 


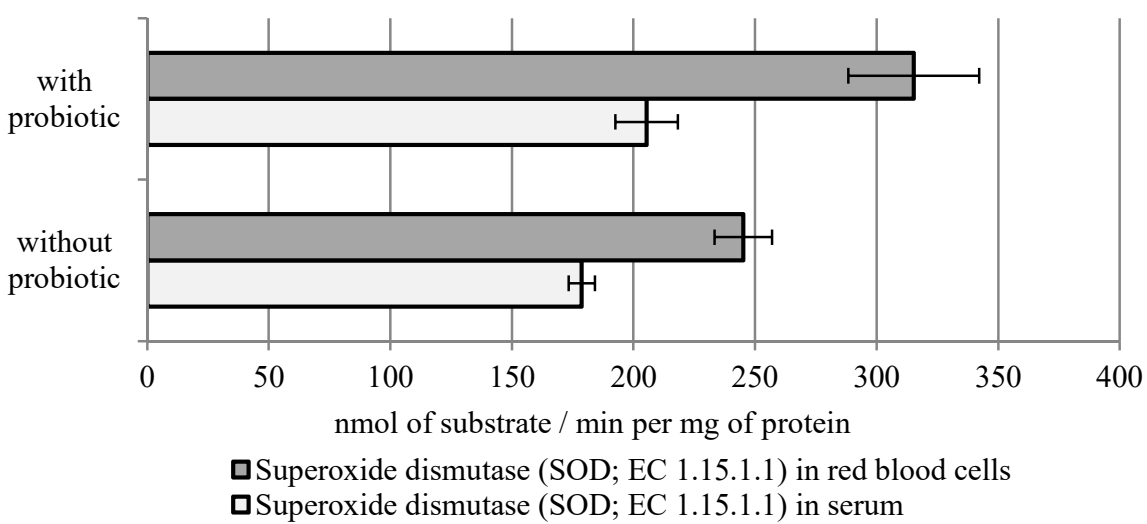

Fig. 5. The activity of superoxide dismutase in serum and red blood cells of catfish.

In catfish grown using microorganism cultures Bacillus subtilis and Bacillus licheniformis the activity of superoxide dismutase in blood serum increased to $205.4 \mathrm{nmol}$ of substrate / min per mg of protein (1.15 times). The activity of superoxide dismutase in erythrocytes against the background of probiotics increased by 1.2 times and amounted to $315.3 \pm 26.9 \mathrm{nmol}$ of substrate / min per mg of protein (fig. 5).

According to the study's results, the introduction of probiotic in fishes feed led to an increase in the activity of catalase to a greater extent than the activity of superoxide dismutase.

Catalase level in erythrocytes of African catfish treated with spore forms of microorganisms Bacillus subtilis and Bacillus licheniformis, amounted to $5.1 \mathrm{nmol}$ of substrate / min per mg of protein, which is significantly higher than the level of $1.37 \mathrm{nmol}$ of substrate / min per $\mathrm{mg}$ of protein in fishes grown with the addition of probiotic microorganisms.

The same regularity is also characteristic of the superoxide dismutase enzyme, which level in erythrocytes against the background of probiotic was $315.3 \mathrm{nmol}$ of substrate / min per mg of protein, and at grown on traditional feed without adding microorganism cultures it was $245.2 \mathrm{nmol}$ of substrate / min per $\mathrm{mg}$ of protein. The revealed phenomenon may indicate the activation of the antioxidant defense system enzyme link in the catfish organism, grown using strains of probiotic microorganisms.

\section{Discussion}

At the present stage of industrial aquaculture development, even in high-tech fish farming systems, it is impossible to create a natural habitat for fish. The artificial habitat necessarily causes a state of stress, primarily due to the high landing density, oxygen deficiency or the inability to find shelter in case of exacerbation of interpersonal relations between members of fish communities.

It was important to find informative indicators that could be indicative in assessing the stress level in the fish organism. First of all, stress hormone cortisol attracted our attention. Study of the cortisol content in fishes showed that when grown in closed CWS, the level of this hormone in fishes is quite high and needs to be reduced.

To achieve this goal, there was used the sporothermin probiotic, consisting of spore forms of microorganisms Bacillus subtilis and Bacillus licheniformis. The probiotic was introduced into fish feed and catfish was grown on such feed for three months. In fishes grown using probiotic, the stress hormone level was determined. It was found that against 
the background of the use of microorganism's cultures Bacillus subtilis and Bacillus licheniformis the cortisol level decreased three times, compared with the original.

The use of micronucleus test showed that a high level of cortisol is accompanied by a high level of micronuclei in peripheral blood erythrocytes and leads to disruption of cell homeostasis.

The micronuclear test was used as an indicator of the organism state at the cellular level [25]. As a result, it was found that a high level of cortisol in the fish organism was accompanied by destructive changes in the nuclear apparatus in a certain proportion of peripheral blood of catfish erythrocytes.

Micronuclei are rounded, relatively small structures consisting of chromatin, which is in the cytoplasm in an interphase state [26-27]. Micronuclei in its structure are similar to the structure of cell nucleus, but differ significantly in size. The composition of micronuclei may include whole chromosomes or chromosome fragments [26]; micronuclei arise when aberrant replacing chromatid structures, around which a shell forms, from the nucleus into the cytoplasm. This process occurs in mitosis telophase. [26, 27]. Most often, the formation of micronuclei occurs when the process of chromosome divergence in mitosis anaphase slows down [26, 27].

In the mitosis anaphase, sister chromatids move to the poles of the cell due to the reduction of tubiline in the microtubules of the spindle and the stretching of sister chromatids formed when the centromere of chromosomes ruptures in the equator and forms a metaphase plate. In the normal fission process, one chromatid moves to one pole, the other moves to another. If microtubules of the fission spindle are disturbed, chromatid nondisjunction occurs and lagging chromatid forms, it does not enter the nucleus of the newly formed cell and leads to the formation of a micronucleus. Thus, the formation of micronuclei occurs from retarded chromatids, which are located in the mother cell or nucleus fragments that occur during mitosis; or from chromosomes derived into mini-cells, followed by its fusion with daughter cells [28]. In cells containing micronuclei, dysfunction occurs due to the activation of protective mechanisms of DNA replication or repair. Cells with micronuclei can enter apoptosis or form binuclear cells [29].

The following indicative indicators for oxidative stress were antioxidant defense enzymes. Against the background of the use of spore forms of microorganisms Bacillus subtilis and Bacillus licheniformis, which were introduced into the feed composition, an increase in antioxidant enzymes, catalase and superoxide dismutase, was noted in the fish organism.

An antioxidant system functions in the fish organism, consisting of enzymatic and nonenzymatic units that bind radicals, prevent the formation or destroy peroxides. Among the most important elements of the fish antioxidant defense is superoxide dismutase and catalase.

Superoxide dismutase is a group of metal enzymes that accelerate the dismutation reaction of superoxide radical anions, maintaining its low concentration in the cell, due to the sequential reduction and oxidation of variable-valence metal ions in the active enzyme center.

Catalase is a heminous enzyme with a molecular weight of $250 \mathrm{kDa}$, contained in peroxisomes, which has a reversibly changing iron ion and four subunits. The enzyme decomposes hydrogen peroxide into molecular oxygen and water [22].

The main role of catalase in fish cells is the destruction of hydrogen peroxide, which is formed in cells due to the oxidases action, and also provides protection against hydrogen peroxide, which can destroy cellular structures.

Against the background of using spore forms of microorganism's cultures with probiotic properties in fish feeding Bacillus subtilis and Bacillus licheniformis and providing partial compensation for stress, cell homeostasis rates have improved. 


\section{Conclusions}

When assessing the cytogenetic homeostasis of fish grown under CWS, there were obtained results, indicating that probiotic microorganisms introduced with food to reduce the cortisol level in the fish blood and the frequency of cell formation with micronuclei in peripheral blood erythrocytes of catfish. The micronuclear test showed a decrease in the percentage of erythrocytes with destructive changes in the nucleus in fish fed with food containing spore forms Bacillus subtilis and Bacillus licheniformis. This is due to a decrease in the damaging effect on the mitotic process of the stress hormone cortisol, the level of which also decreased under the probiotic influence.

Micronuclear test can be used as an indicator of catfish cell homeostasis. This will allow timely formation of a prognostic assessment and early diagnosis of the pathological process development in the fish organism grown in industrial aquaculture.

Research results showed that microorganisms Bacillus subtilis and Bacillus licheniformis, included in the probiotic, activate the enzyme link of the catfish antioxidant system. It can be assumed that the metabolites of probiotic microorganisms' strains contribute to a decrease in the level of toxic products formed during lipid peroxidation. In practice, this protects the organs and tissues of catfish reared in industrial aquaculture from oxidative damage.

The obtained results characterizing the state of fish cell homeostasis in industrial aquaculture are new.

The use of a probiotic based on microorganisms' spore forms Bacillus subtilis and Bacillus licheniformis provides stress reduction for catfish, opening up prospects for correcting the negative effects generated by high-tech fish farming systems.

Summarizing the foregoing, we can conclude that the use of microbiota with probiotic properties Bacillus subtilis and Bacillus licheniformis contributes to the antioxidant protection activation, stress protection of catfish (Clarias gariepinus), provides cytogenetic homeostasis. Microorganisms with the properties of probiotics can provide an increase in the efficiency of industrial aquaculture and its progress.

\section{References}

1. E.M. Romanova, V.N. Lyubomirova, V.V. Romanov, M.E. Mukhitova, T.M. Shlenkina, Egyptian journal of aquatic research 44(4), 315-319 (2018)

2. E. Spirina, E. Romanova, V. Romanov, V. Lyubomirova, L. Shadyeva, T. Shlenkina, L. Rakova, IOP conference series: Earth and environmental science 403, 012220 (2019)

3. M. Bentzon-Tilia, E.C. Sonnenschein, L. Gram, Microbial biotechnology 9(5), 576584 (2016)

4. H. Ghomrassi, O.B. Braiek, K. Hani, T. Ghrairi, Y. Choiset, T. Haertlé, J.-M. Chobert, Diseases of aquatic organisms 118(1), 31-43 (2016)

5. X. Xiong, G. Huang, Y. Peng, X. Liu, Journal of fisheries of China 40(1), 73-82 (2016)

6. M.E. Yonar, S.M. Yonar, M.Z. Çoban, M. Eroğlu, Environmental toxicology 29(2), 155-164 (2014)

7. I.I. Rudneva, Comparative biochemistry and physiology. part C: pharmacology, toxicology and endocrinology 118(2), 255-260 (1997)

8. E. Calcagno, P. Durando, M.E. Valdés, L. Franchioni, M.D.L.T. Bistoni, Physiology \& Behavior 158, 68-75 (2016) 
9. E. Kulczykowska, H. Kalamarz-Kubiak, M. Gozdowska, E. Sokołowska, Comparative Biochemistry and Physiology. Part A: Molecular \& Integrative Physiology 218, 1-7 (2018)

10. M. Fenech, Mutation Research 455, 81-95 (2000)

11. J. Mersh, M.N. Beauvais, P. Nagel, Mutation Research 371, 47-55 (1996)

12. F.J. Gatesoupe, Bioactive Foods in Promoting Health, 541-552 (2010)

13. V.A. Zuenko, K.S. Laktionov, I.V. Pravdin, L.Z. Kravtsova, N.A. Ushakova, Journal of Ichthyology 57(1), 152-157 (2017)

14. B. Thankappan, D. Ramesh, S. Ramkumar, K. Anbarasu, K. Natarajaseenivasan, Applied Biochemistry and Biotechnology - Part A Enzyme Engineering and Biotechnology 175(1), 340-353 (2015)

15. J. Kazarjan, M. Vaher, M. Kulp, M. Kaljurand, T. Hunter, G.J. Hunter, R. Bonetta, D. Farrugia, Journal of separation science 38(6), 1042-1045 (2015)

16. Y. Kaminsky, E. Kosenko, G. Aliev, Cardiovascular and hematological agents in medicinal chemistry 14(1), 49-52 (2016)

17. M.E. Yonar, Ecotoxicology and environmental safety 151, 49-54 (2018)

18. M.E. Yonar, Fish \& shellfish immunology 32(6), 994-1001 (2012)

19. L.T. Gressler, F.J. Sutili, E.M.H. Saccol, T.S. Pês, T.V. Parodi, M.A. Pavanato, B. Baldisserotto, L. Loebens, S.T. Da Costa, Aquaculture research 47(7), 2297-2306 (2016)

20. F.N. Ekeh, E.N. Ekechukwu, C.I. Atama, F.I. Ezenwajiaku, C.M. Ohanu, J.I. Nzei, I.O.N. Aguzie, G.E. Odo, U.M.E. Dibuah, African journal of aquatic science 43(4), 393-403 (2018)

21. H. Sadauskas-Henrique, M.M. Sakuragui, M.G. Paulino, M.N. Fernandes, Environmental monitoring and assessment 181(1-4), 29-42 (2011)

22. T. Wang, Y. Cheng, X. Chen, Z. Liu, X. Long, Chinese journal of oceanology and limnology 35(1), 89-97 (2017)

23. X. Xiong, Y. Huang Peng, X. Liu, Journal of Fisheries of China 40(1), 73-82 (2016)

24. A. Taheri Mirghaed, M. Ghelichpour, S.M. Hoseini, Fish \& Shellfish Immunology 81, 182-188 (2018)

25. F.D. Gökalp Muranli, U. Güner, Genetic Toxicol. and Environ. Mutagenesis 726, 104 $-108(2011)$

26. M. Witczak, I. Kociszewska, J. Wilczynski et al., Mutation Research 701(2), 111-117 (2010)

27. A.S.A. Harabawy, Th.A. Ahmed, Ecotoxicology and Environmental Safety 103, 61-67 (2014)

28. J. Katic, E. Cemeli, A. Baumgartner et al., Food and chemical toxicology 48(10), 2612-2623 (2010)

29. K-i. Utani, Y. Kohno, A. Okamoto et al., PLoS ONE 5(4) (2010) 